\title{
Modeling the epidemic dynamics of COVID-19: Agent-based approach including molecular dynamics simulation and SEIR type methods
}

Amir Lohrasebi ( $\square$ lohrasebi@nano.ipm.ac.ir)

University of Isfahan

Fatemeh Aghaei

University of Isfahan

\section{Research Article}

Keywords: COVID-19, Epidemiology modeling, SEIR model, Molecular dynamics model

Posted Date: November 11th, 2020

DOI: https://doi.org/10.21203/rs.3.rs-105131/v1

License: (c) (i) This work is licensed under a Creative Commons Attribution 4.0 International License.

Read Full License

Version of Record: A version of this preprint was published at International Journal of Modeling, Simulation, and Scientific Computing on November 11th, 2020. See the published version at https://doi.org/10.1142/S1793962321500574. 


\title{
Modeling the epidemic dynamics of COVID-19: Agent-based approach including molecular dynamics simulation and SEIR type methods \\ F. Aghaei, A. Lohrasebi* \\ Department of Physics, University of Isfahan, Isfahan, 8174673441, Iran.
}

\begin{abstract}
In this study we developed a SEIR model, including social interactions and individual human mobility in everyday activities. For this purpose, daily mobility of people was considered by using the molecular dynamic method and the virus spreading was modeled employing the ordinary SEIR scheme. Utilizing this model, the variation of population size, density, and health strategy as well as the effect of busy places such as malls, were considered. The results show that, our flexible model is able to consider the effects of different parameters such as distance between peoples, local population density and health strategy in the outbreak.
\end{abstract}

Keywords: COVID-19; Epidemiology modeling; SEIR model; Molecular dynamics model.

*Corresponding Author: lohrasebi@phys.ui.ac.ir, Tel/: (+98) 3137934727, Fax: (+98) 3137934800

\section{Introduction}


Virus epidemics or outbreaks happened by the viruses, have been wide spreading among human societies. Usually, one of the most important ways of the virus spreading is the people's daily life close contact, which may cause one healthy person to be faced with an infected one and inhales very small droplets produced by him/her (possibly created by talking, coughing, or sneezing). In December 2019 a novel type of coronavirus, called COVID-19, was identified in Wuhan-China, which has rapidly spread all around the world due to the accessibility of global travel and close contact of people [1]. This virus, COVID-19, is a new one, and in addition to its biological harm issues, many of its epidemic details are still unclear and under investigation. Regarding this situation, some public health strategies, like maintaining overall personal hygiene and social distancing, are recommended to cut off the infection transmission chain. Moreover, high-risk activities such as being in crowded places, like busy restaurants or shopping centers, and travelling on public transport, are controlled seriously to reduce the risk of the virus spreading.

Considering these conditions, the theoretical modeling of disease outbreak could be helpful to select more efficient policies and actions for controlling the outbreak in realistic cases. In the recent years, agent-based models (ABMs) have been extensively used for modeling such pandemics [2-12]. Some of the basic and simplest epidemiological models which are widely used to predict these kinds of epidemic diseases are SIR type models (SIR, SEIR and SEIRD models) [7-14] that represent the disease timeline through three, four and five status, where the variables S, E, I, R and D denote the number of individuals in susceptible, exposed, infected, recovered, and deceased compartment states, respectively. In this type of classical epidemiological models, individual real-life information is not considered and hence these models are limited to describe the details of outbreaks completely. Recently, to obtain better descriptions of the disease spreading, agent-based models which include the individual's behavior were employed for modeling the virus spreading. To this end, combination of the methods was used to predict the outbreak dynamics, in some investigations. As an example, Kie et al. presented a hybrid model for COVID-19 pandemic prediction based on a SEIR approach and the ABM Monte Carlo simulation [15]. In another study, Martınez et al. [16] planned an $\mathrm{ABM}$ system to simulate virus spreading by means of agents that are characterized by their individual mobility patterns and social networks from cell phone records to model the virus spreading. It is well known that useful models are based on simplifying assumptions, however data obtained from all models are some kinds of estimations, but they can be useful if their basic assumptions are good enough for the particular applications. We believe that more realistic models than the ordinary existing ones 
such as the normal forms of SEIR family models are needed to describe the virus epidemic in detail, due to some noticeable features of COVID-19 outbreak affected by the public situations such as social culture, local medical resources, health strategies and individual activities. Thus, in this study we aimed to improve the SEIR approach by considering the people daily mobility and communications. To this end, the molecular dynamic (MD) simulation and ordinary SEIR epidemic methods were combined, in which people mobility was considered using the MD simulation method, and the virus spreading between people was modeled employing the SEIR scheme. The method tracks the individual human mobility during the epidemic timeline, by time interval less than an hour. So, it not only considers the daily routine activities of each person in the virus spreading, but also allows us to add the effects of geometries of public sites and places, like malls in virus epidemic. In addition, this flexible model lets us to consider the effects of impressive parameters on the outbreak, such as variation of distance between two individuals. Moreover, this method makes it possible to understand that how the usage of suitable public health strategies can effectively control and prevent the transmission of these kinds of communicable diseases. This approach can be especially useful to establish the efficient policies to control such diseases, for cases such as universities, schools, government buildings and so on, mainly after the post lockdown phase of the outbreak. Hence, we believe that employing this simple model, based on well-defined assumptions can provide a practical approach to overcome the two important features of COVID-19 mentioned above. In addition, this scheme can be used to model other kinds of similar infectious disease, and also comparable complex systems such as animal herd dynamics and communication in social networks, by utilizing the proper parameters.

\section{Materials and Method}

We model the epidemiology of COVID-19 outbreak employing an ABM based on combination of the MD simulation method and the ordinary SEIR model. In our improved model, the population was represented by a collection of $\mathrm{N}$ particles (each person considered as a particle), in which the people mobility was simulated using the MD simulation method and virus spreading between the people was modeled utilizing SEIRD method with the five mentioned compartments.

In fact, we used the MD simulation to create the network dynamics of contacts between the individuals. For this purpose, each person was considered as a particle in the simulation box, and the interactions between people (particles) in the society were calculated from the simple form of the Lennard-Jonze potential as follows: 


$$
E_{L J}^{S}=\varepsilon\left[\left(\frac{\delta}{r_{i j}}\right)^{2}-\left(\frac{\delta}{r_{i j}}\right)^{4}\right],
$$

where $\varepsilon$ and $\delta$ are respectively related to the potential magnitude and the equilibrium distance between the particles and $r_{i j}$ is the distance between the $\mathrm{i}$-th and $\mathrm{j}$-th particles. The equations of motion were integrated via the application of Velocity-Verlet method [17]. To change the social conditions such as social distancing, the related simulation parameters must be changed, accordingly. For example, the parameters $\delta$ (potential distance cut-off) and infectious rates, are related to the social distancing strategies. In the simulations at first, the particles are allowed to interact with each other and be in motion accordingly, during each MD step. After each step, the SEIR method algorithm was used to model the virus spreading among the people. As it is known, when the virus introduced to a population by some infected ones, the people status switched from one of the mentioned compartment to the next one, i.e. $S \rightarrow E \rightarrow I \rightarrow R$, and with small probabilities from $E$ and $I$ to $D$. The variation of population compartments were computed during the time, governed by a set of five coupled differential equations, which are presented in Eq. (2).

$\frac{\mathrm{d} S}{\mathrm{dt}}=-\beta_{1} \frac{S I}{N}-\beta_{2} S$

$\frac{d E}{d t}=\beta_{1} \frac{S I}{N}+\beta_{2} S-\alpha E-\sigma_{1} E$

$\frac{d I}{d t}=\alpha E-\gamma I-\sigma_{2} I$

$\frac{d R}{d t}=\gamma I$

$\frac{d D}{d t}=\sigma_{1} E+\sigma_{2} I$

Where $\mathrm{S}, \mathrm{E}, \mathrm{I}, \mathrm{R}$ and $\mathrm{D}$ denote the above mentioned populations, and $\mathrm{N}=\mathrm{S}+\mathrm{E}+\mathrm{I}+\mathrm{R}+\mathrm{D}$ is the total population. The parameters $\beta_{1}, \beta_{2}, \alpha, \gamma, \sigma_{1}$ and $\sigma_{2}$ are the transition rates between the five compartments and are $\alpha=1 / \mathrm{A}, \beta_{1}=1 / \mathrm{B}$ and $\gamma=1 / \mathrm{C}$, where $\mathrm{A}, \mathrm{B}$ and $\mathrm{C}$ are the latent, contact, and infectious periods. Among these parameters, $\mathrm{A}$ and $\mathrm{C}$ are interpreted as the disease specific and $B$ as the behavior specific. It is necessary to mention that the parameter $\beta_{1}$ is related to the inverse of distance between the two particles, as well, i.e. the larger the distance between $\mathrm{S}$ and I ones, the less the probability of translation from $\mathrm{S}$ to $\mathrm{E}$ status. Furthermore, $\beta_{2}$ is the environmental transition coefficient from $S$ to $E$, i.e. the probability of getting the 
virus from the environment, indirectly. The parameter $\sigma_{1}$ and $\sigma_{2}$ denote the mortality rate of disease, at the exposed and infectious levels.

This set of equations was applied as a stochastic process to model the disease transmission in $\mathrm{ABM}$. To this end, according to the possible disease progression of an individual, a susceptible one may become exposed if he/she directly is faced with an infectious one at rate $\beta_{1}$ (which could be related to the distance) or may become exposed indirectly by inhaling the infected droplets floating in his/her surrounding at rate $\beta_{2}$.

These probability parameters can be decreased considering suitable public health strategies such as facial masking and frequent hand-washing. The exposed one switches to the infected one after 3 days (with the rate of $\alpha=1 / 3 \mathrm{Day}^{-1}$ ), and the infected one to the recovered one after 15 days (with the rate of $\beta=1 / 15 \mathrm{Day}^{-1}$ ) [8]. In addition, the exposed and infected ones are removed from the active population at the rates $\sigma_{1}$ and $\sigma_{2}$. To consider the effect of especial busy places, such as malls, the dynamics of particles in the system were simulated in the present of an attractive force. In this way, a Gaussian function form was chosen as the attraction force as bellow:

$$
F_{A t t}^{S}=F_{0} \exp \left[-\left(r_{i}-r_{0}\right)^{2} /(2 a)\right],
$$

Where $F_{0}$ is the force intensity, $a$ is the width of the potential, $r_{0}$ is position of the center of the place and $r_{i}$ is the location of individual $i$. The lockdown, and closure or limitation of the public places' services are reflected on $F_{0}$ and $a$ parameters. The parameters values used in our computational model are listed in table 1.

Table (1) List of parameters and their values used in our simulations [8,11].

\begin{tabular}{|c|c|c|c|}
\hline parameter & Description & unit & value \\
\hline $\boldsymbol{\alpha}$ & Latency rate & Day $^{-1}$ & $0.33(1 / 3)$ \\
\hline $\boldsymbol{\beta}_{\mathbf{1}}$ & Infection rate (direct coefficient form infected \\
one) & Day $^{-1}$ & 0.067 \\
\hline $\boldsymbol{\beta}_{\mathbf{2}}$ & Infection rate (direct coefficient form \\
environment) & Day $^{-1}$ & 0.0067 \\
\hline $\boldsymbol{\gamma}$ & Recovery rate & & \\
\hline $\boldsymbol{\sigma}_{\mathbf{1}}$ & Death rate (for exposed compartment) & Day $^{-1}$ & $0.047(1 / 21)$ \\
\hline $\boldsymbol{\sigma}_{\mathbf{2}}$ & Death rate (for infected compartment) & Day $^{-1}$ & 0.0001 \\
\hline $\boldsymbol{\delta}$ & Length parameter of LJ potential & $\mathrm{m}^{-1}$ & 0.5 \\
\hline $\boldsymbol{\varepsilon}$ & Energy parameter of LJ potential & $\mathrm{J}$ & 2 \\
\hline $\mathbf{F}_{\mathbf{0}}$ & Attractive force coefficient & $\mathrm{N}$ & 15 \\
\hline
\end{tabular}




\begin{tabular}{|c|c|c|c|}
\hline $\mathbf{N}$ & Number of population & - & $1500-2600-5200$ \\
\hline $\mathbf{a}$ & Gaussian potential width & $\mathrm{m}^{2}$ & 10000 \\
\hline
\end{tabular}

\section{Results and Discussion}

The aim of this investigation is to predict the outbreak scenarios by using our developed model, based on MD and SEIR methods. As it was mentioned, in our proposed epidemiological model, the people mobility was simulated employing the MD simulation approach and the virus spreading between the people was modeled using SEIR method, at each MD time step. Regarding this approach, we considered the effect of social distancing, individuals' mobility and public health strategies such as social distancing, on the diseases outbreak. To this ends, several simulations were performed to examine the influence of some parameters, such as the system size and physical distancing, to prevent the transmission of the disease and to maintain the public health. In all simulations, the initial numbers of susceptible, exposed, infected, recovered, and deceased individuals were set at N-1, 0, 1, 0 and 0 , respectively. The initial arrangement of the number of 2600 people in the square simulation box of $1000 \times 1000 \mathrm{~m}^{2}$ is shown in Fig. 1-a.

\section{Figure (1)}

In the first computational examination, we considered the influence of the total population number on the outbreak dynamics. To this end, three population numbers was chosen, i.e. 1500, 2600 and 5200 persons, with the same population density. The variations of the five populations during the time for the three mentioned total population numbers are shown in the panels of Fig. 2. From these curves, as we expected from SEIR model, it can be seen that as the susceptible population decreases over time, the exposed population and after it the infected population first grow up, then reach a peak, then begin to decline and eventually reach zero. Furthermore, it can be observed that by decreasing the infected population, the recovered and the deceased populations increase toward their final values.

\section{Figure (2)}

As can be seen from Fig. 2-a, 2-b and 2-c, the dynamics of population compartments are nearly similar for three population numbers, and only the maximum picks values are different according to the population. In addition, it is found that nearly all people have experienced the exposed level, and their status changed to the recovered or death during about 50 days. Moreover, the population variation of E, I, R and D compartments per day were calculated and shown in the panels of Fig. 2-d, 2-e and 2-f, for $\mathrm{N}=1500,2600$ and 5200, respectively, 
which their variation are very similar for three cases. These curves can be useful to predict the second phase of the outbreak, i.e. if after the first negative or zero values of $\Delta \mathrm{I}$, the positive values are observed again, the second phase of outbreak may be started. To clear the effect of the people mobility simulated by MD, a set of three similar mathematical SEIR modeling were performed on a system, in which individuals were fixed on a square lattice network and they could interact with their first and second-nearest neighbors, for the mentioned populations, i.e. 1500, 2600 and 5200 ones. As it is presented in the panels of Fig. 3 , the results are nearly similar but for a larger infectious rate $\left(\beta_{1}=0.125, \beta_{2}=0.025, \sigma_{1}=0.005\right.$, $\left.\sigma_{2}=0.025\right)$. So, considering the people mobility in the model caused to increase the virus spreading rate. It is necessary to mention that the simulation on the fixed networks was faster and can be used for bigger population, but our model is more flexible and considers more details as well as people motility, as it will be shown in the following.

\section{Figure (3)}

Therefore, it was achieved that population number is not the key parameter in the outbreak if the population densities are the same, i.e. it seems that the population density can be the important parameter in the outbreak dynamics and to test this propose, three amounts of population number densities were examined and the obtained results are presented in panels of Fig. 4. From this figure, it is observed that the rate of outbreak dynamics was reduced for the smaller population density, i.e. the duration period of the disease in the chosen society increased for lower density. It is due to the fact that by decreasing the density, the average amount of separation distance between the people increased and so the probability of facing with the sick person was reduced. Thus, as it was known, reducing the people density in the public places can be a successful strategy for controlling the outbreak, and we believe our method can be useful to determine the optimal people density in small public places such as schools and universities.

\section{Figure (4)}

To consider the effect of public health strategies, such as social distancing, we increased the average distance between the people by increasing $\delta$ in L-J potential value. Furthermore, the infected persons were not allowed to interact with the other people by decreasing $\beta_{1}$ value, as quarantine. In addition, the probability of getting the virus from the environment is reduced by reducing $\beta_{2}$ as the application of some health strategies such as facial masking and hand washing. The obtained results shown in Fig. 5 imply that applying these health strategies caused to increase the outbreak period (more than 250 days) in comparison with the condition that these strategies were not applied (less than 50 days). As can be seen in Fig. 5 the 
maximum number of sick people (about 0.1 of publication for the case of 2600 persons) is less than the same value when the public-health strategies have not been applied (about 0.8 of publication for the case of 2600 persons). Accordingly, the ailing persons can be better supported by limited public medical resources (usually in good condition, the public medical resources are enough for 0.1 of publication), which leads to reduction in the deceased population. Hence, the risk of population's health harming from the disease will be reduced by increasing the outbreak period.

\section{Figure (5)}

Finally, in order to investigate the effect of special crowded public places such as malls, transport stations and so on, we added the attracting force of form Eq. (3), in the MD simulation which is caused to increase the local population density in that place (as it is shown in Fig. 1-b. The result of this simulation is presented in Fig. 6. As can be seen adding a public place in the system caused to increase the infection rate and hence decreases the outbreak duration time. Declining in the outbreak duration leads to increase the public damage, as it is described in the previous section, i.e. the maximum number of daily sick people is higher when the attraction public place was added in the model. So the public medical resources probably cannot support the numerous people in the small time duration, which leads to increase the health-damaged risk.

\section{Figure (6)}

Therefore, as it was shown, this flexible model could be very useful for investigating such pandemic cases, and also in modeling the information diffusion in the social communication networks and social face-to-face interaction between individuals, animal behaviors within a herd and so on.

\section{Conclusion}

In this paper we have modified the SEIR approach by considering the people daily activities to simulate an outbreak dynamics. To this end, a classical MD simulation method was utilized to simulate the people mobility and SEIR model was employed for modeling disease spreading among the people. To examine the impact of various public-health strategies, such as physical distancing, on the resulting epidemic spreading, several simulations were performed. Our model has confirmed that the number of people in a system is not a significant factor in the outbreak while the population density is an effective parameter in the virus spreading. We have also realized that as far as the people mobility is concerned, the disease spreading rate is more than the situation of a network of fixed people in which every 
individual has limited neighbors. Furthermore, our simulations show that the application of the social distancing can lead to a reduction in the epidemic spreading by influencing $\beta_{1}$ and $\beta_{2}$ values. So it allows us to determine the optimal physical distance to control the pandemic. As a final point, to concern the effect of crowded public places such as malls, the local population density in such places was increased by adding the attracting Gaussian force in the MD simulation which leads to increase the COVID-19 spreading rate in the system. This approach can be easily adjusted to model such infectious disease that follows the SEIR person to person transmission dynamics, and also other kinds of similar complex systems such as communication in social dynamics networks and animal herd behaviors, by changing the related parameters.

\section{Author contribution statement}

All authors contributed equally to the work.

\section{Declaration of interests}

The authors declare that they have no known competing financial interests or personal relationships that could have appeared to influence the work reported in this paper.

\section{References:}

[1] Q. Li, X. Guan, P. Wu, X. Wang, Z. Feng, Early transmission dynamics in Wuhan, China, of novel coronavirus-infected pneumonia, New Engl J Med. 382(13) (2020) 1199-1207.

[2] E. Bruch, J. Atwell, Agent-Based Models in Empirical Social Research, Social Methods Research 44(2) (2015) 186-221.

[3] N. Gilbert, Agent-Based Models. London: Sage Publications (2008).

[4] E. Hunter, B. Mac Namee, J.D. Kelleher, A Taxonomy for Agent-Based Models in Human Infectious Disease Epidemiology, Journal of Artificial Societies and Social Simulation 20(3) (2017) 2.

[5] A.T. Crooks, A.B. Hailegiorgis, An agent-based modeling approach applied to the spread of cholera, Environmental Modelling \& Software 62 (2014) 164-177.

[6] E. Hunter, B. Mac Namee, J. Kelleher, Correction: An open-data-driven agent-based model to simulate infectious disease outbreaks, PloS one 14(1) (2019) e0211245.

[7] J.L. Aron, I.B. Schwartz, Seasonality and period-doubling bifurcation in an epidemic model, J Theor Biol. 110 (1984) 665-679. 
[8] W.T. Zha, F.R. Pang, N. Zhou, B. Wu, Y. Liu, Y.B. Du, X.Q. Hong, Y. Lv, Research about the optimal strategies for prevention and control of varicella outbreak in a school in a central city of China: based on an SEIR dynamic model, Epidemiology and Infection. 148(56) (2020) 1-8.

[9] J. Horn, O. Damm, W. Greiner, Influence of demographic changes on the impact of vaccination against varicella and herpes zoster in Germany-a mathematical modelling study, BMC Medicine 16 (2018) 3-12.

[10] H. Hethcote, M. Zhien, L. Shengbing, Effects of quarantine in six endemic models for infectious diseases, Mathematical Biosciences 180 (2012) 141-160.

[11] M. Peirlinck, K. Linka, F. Sahli Costabal, E. Kuhl, Outbreak dynamics of COVID-19 in China and the United States, Biomech Model Mechanobio (2020) doi:10.1007/s10237-02001332-5.

[12 S. Patrikar, D. Poojary, D.R. Basannar, D.S. Faujdar, R. Kunte, Projections for novel coronavirus (COVID-19) and evaluation of epidemic response strategies for India, Medical journal Armed Forces India 76 (2020) 268-275.

[13] E. Volz, SIR dynamics in random networks with heterogeneous connectivity, Journal of Mathematical Biology 56(3) (2008) 293-310.

[14] K. Linka, M. Peirlinck, F.S. Costabal, E. Kuhl, Outbreak dynamics of COVID-19 in Europe and the effect of travel restrictions, Computer Methods in biomechanics and biomedical engineering 3636 (2020) 1-8.

[15] D. Kai, G.P.h. Goldstein, A. Morgunov, V. Nangalia, A. Rotkirch, Universal Masking is Urgent in the COVID-19 Pandemic: SEIR and Agent Based Models, Empirical Validation, Policy Recommendations (2020).

[16] E. Frıas-Martınez, G. Williamson, V. Frıas-Martınez, An Agent-Based Model of Epidemic Spread using Human Mobility and Social Network Information. In: Privacy, security, risk and trust (PASSAT) and IEEE third inernational conference on social computing (SocialCom), IEEE Press, New York (2011) 57-64.

[17] M.P. Allen, D.J. Tildesley, Computer Simulation of Liquids. Clarendon, Oxford (1987). 


\section{Figure's}

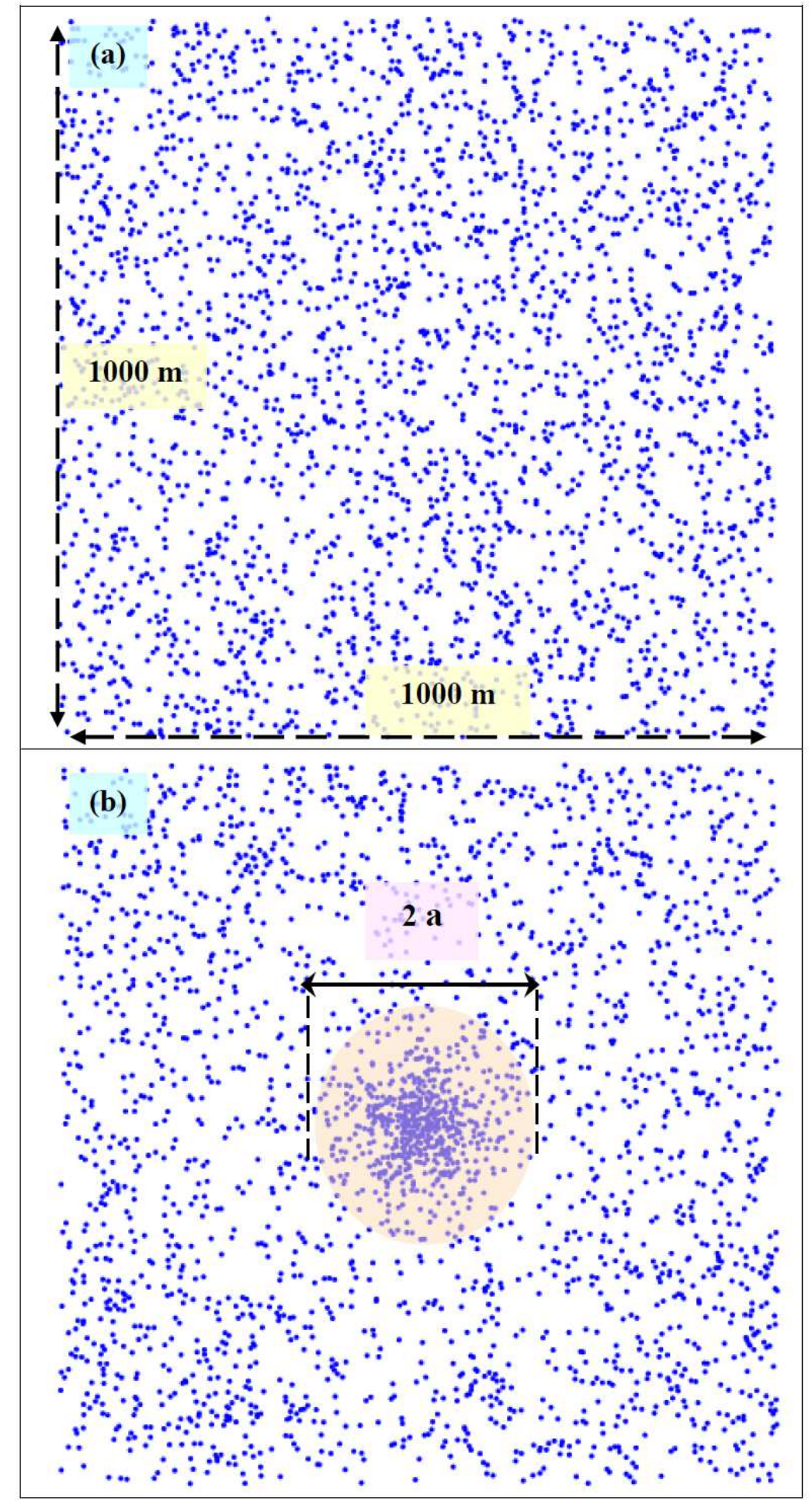

Figure 1: The initial arrangement of the number of 2600 people in the square simulation box of $1000 \times 1000 \mathrm{~m}^{2}$, (a) for the system without the crowded place, (b) for the system without the crowded place in the center of the simulation box. 


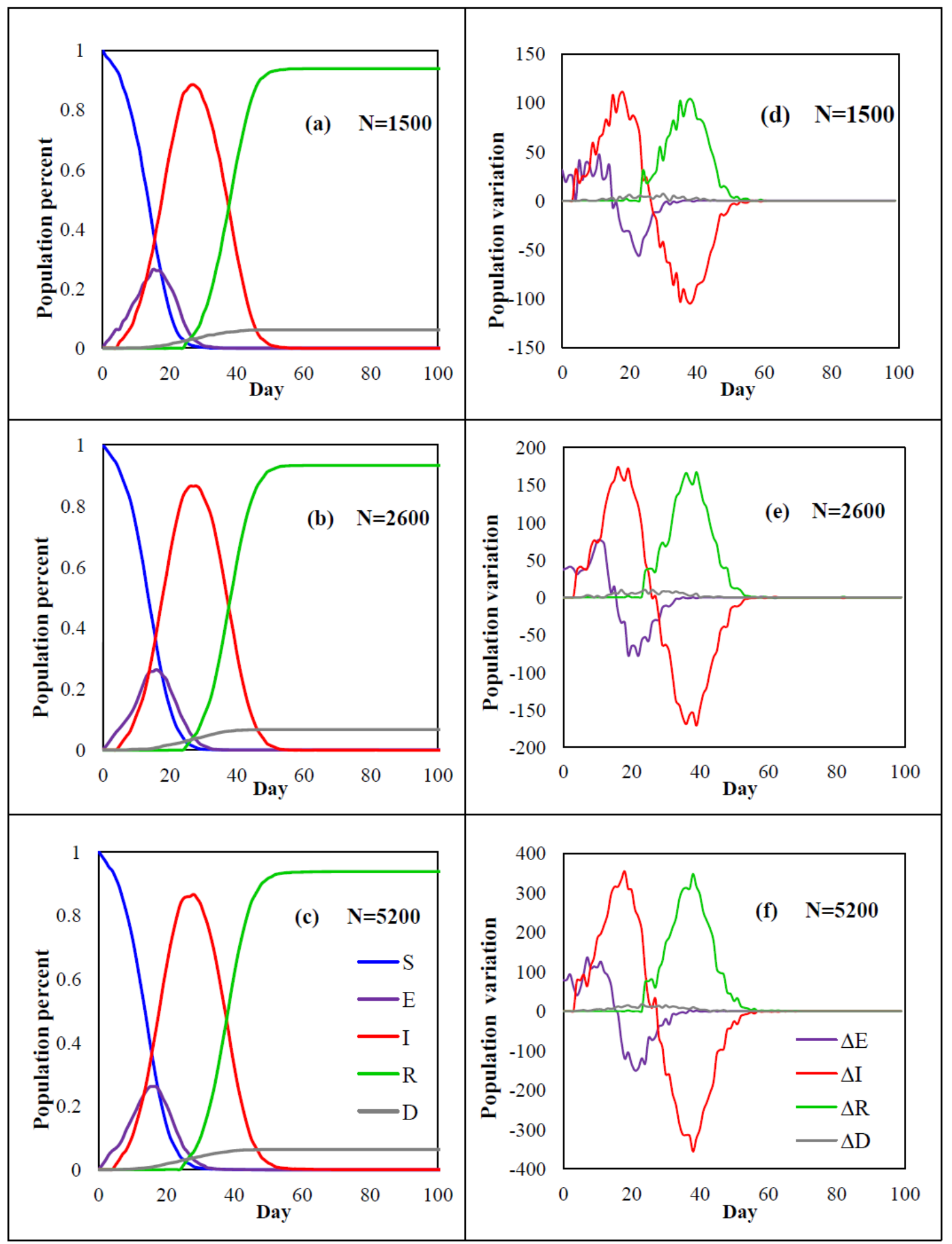

Figure 2: The percentage variations of the five populations during the time for the total population number of, (a) 1500, (b) 2600, and (c) 5200, and the population variation of E, I, $\mathrm{R}$ and D compartments per day for the total population number of, (d) 1500, (e) 2600, and (f) 5200, utilizing our model. 


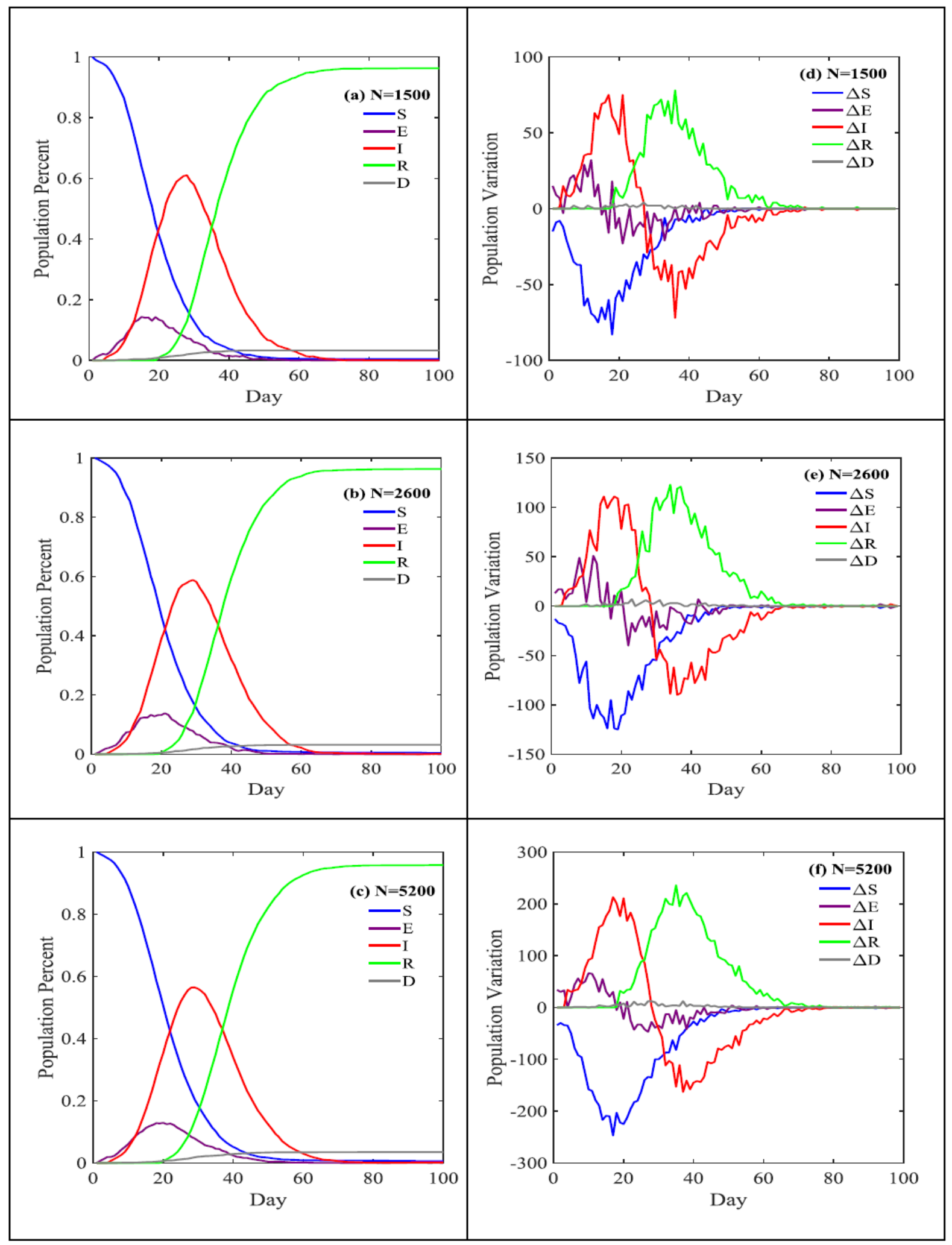

Figure 3: The percentage variations of the five populations during the time for the total population number of, (a) 1500, (b) 2600, and (c) 5200, and the population variation of E, I, $\mathrm{R}$ and D compartments per day for the total population number of, (d) 1500, (e) 2600, and (f) 5200, utilizing classical SEIR model with a fixed square lattice network. 


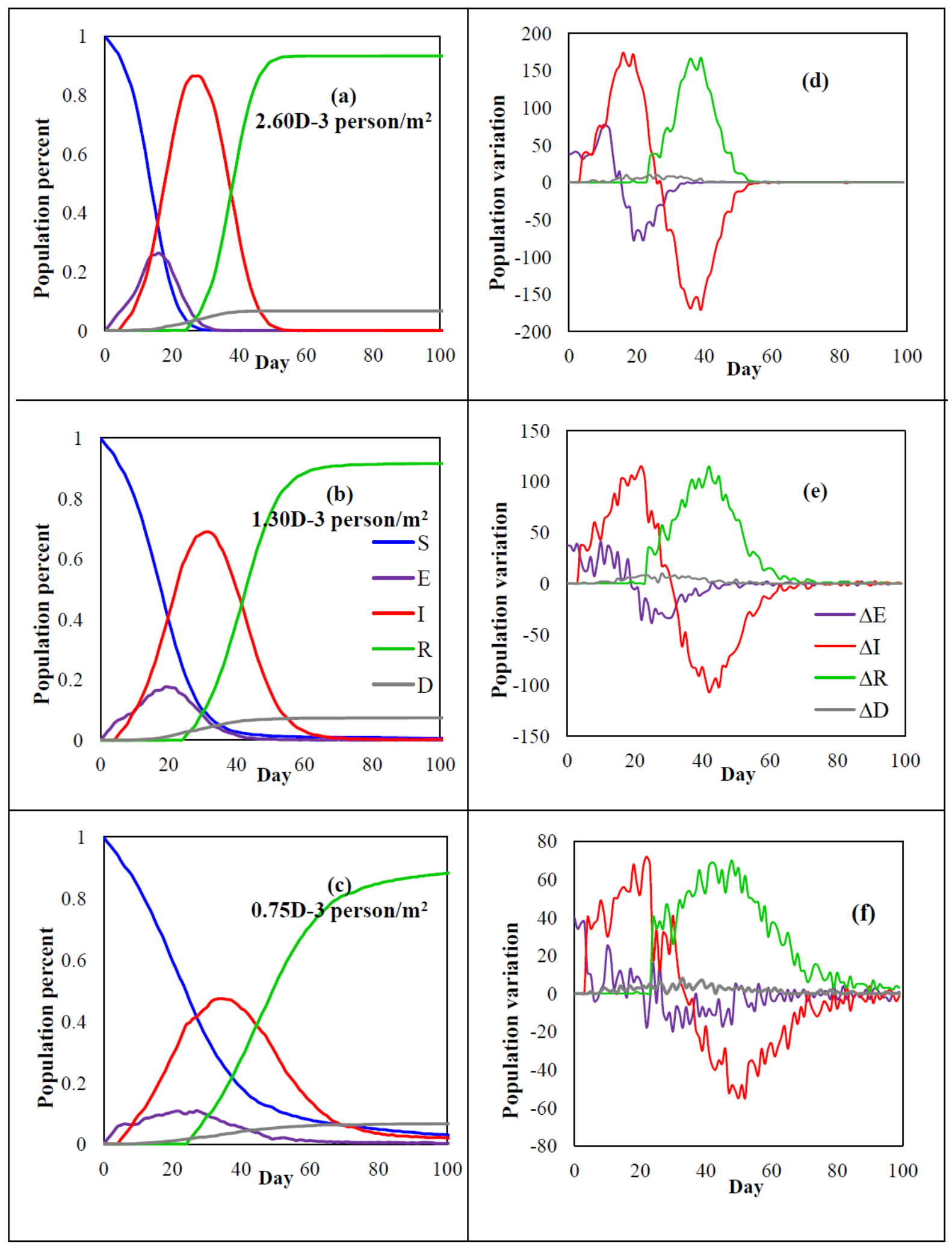

Figure 4: The percentage variations of the five populations during the time for the population number densities of, (a) 2.60D-3, (b) $1.30 \mathrm{D}-3$, and (c) $0.75 \mathrm{D}-3$ person $/ \mathrm{m} 2$, and the population variation of $\mathrm{E}, \mathrm{I}, \mathrm{R}$ and $\mathrm{D}$ compartments per day for the total population number of, (d) 2.60D-3, (e) $1.30 \mathrm{D}-3$, and (f) $0.75 \mathrm{D}-3$ person/m2, utilizing our model. 


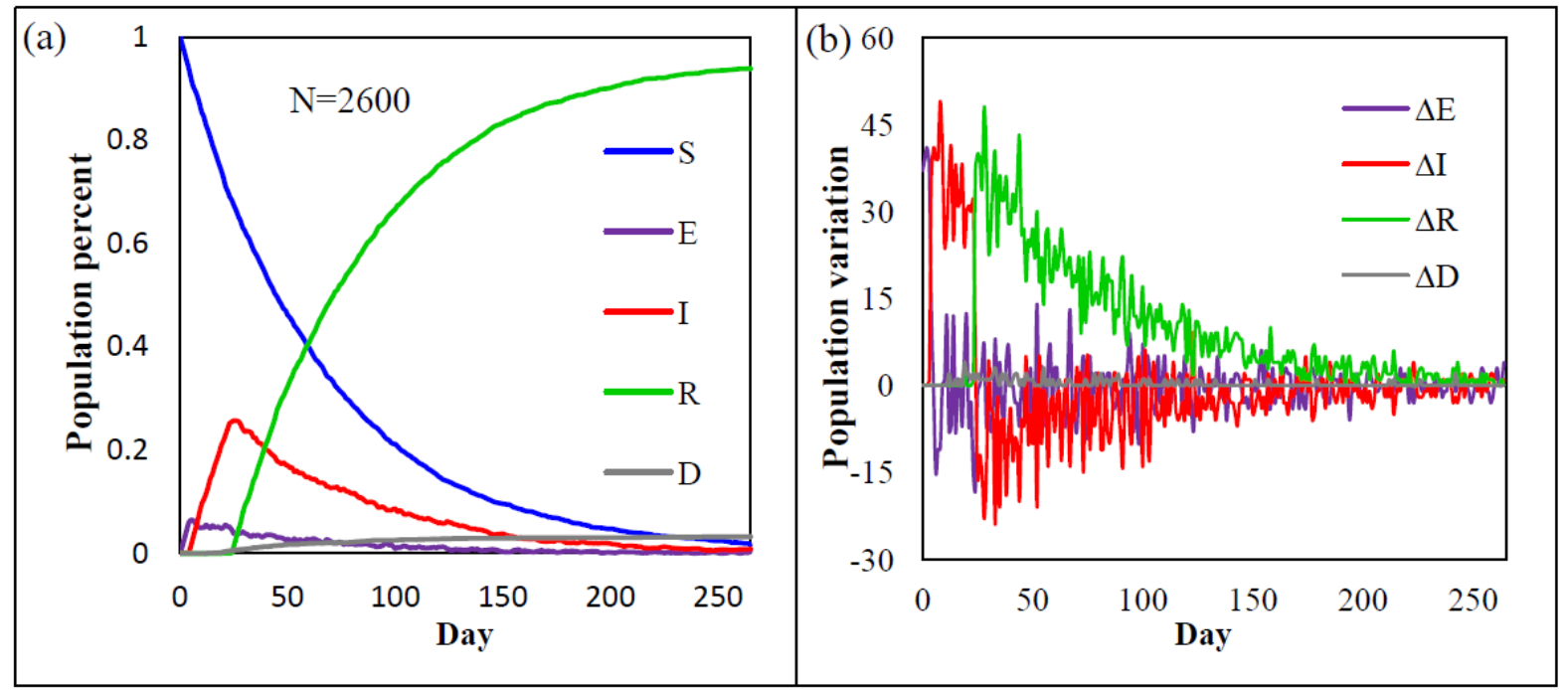

Figure 5: The effect of public health strategies for the total population number of 2600 persons, (a) the percentage variations of the five populations during the time, (b) the population variation of $\mathrm{E}, \mathrm{I}, \mathrm{R}$ and $\mathrm{D}$ compartments per day.
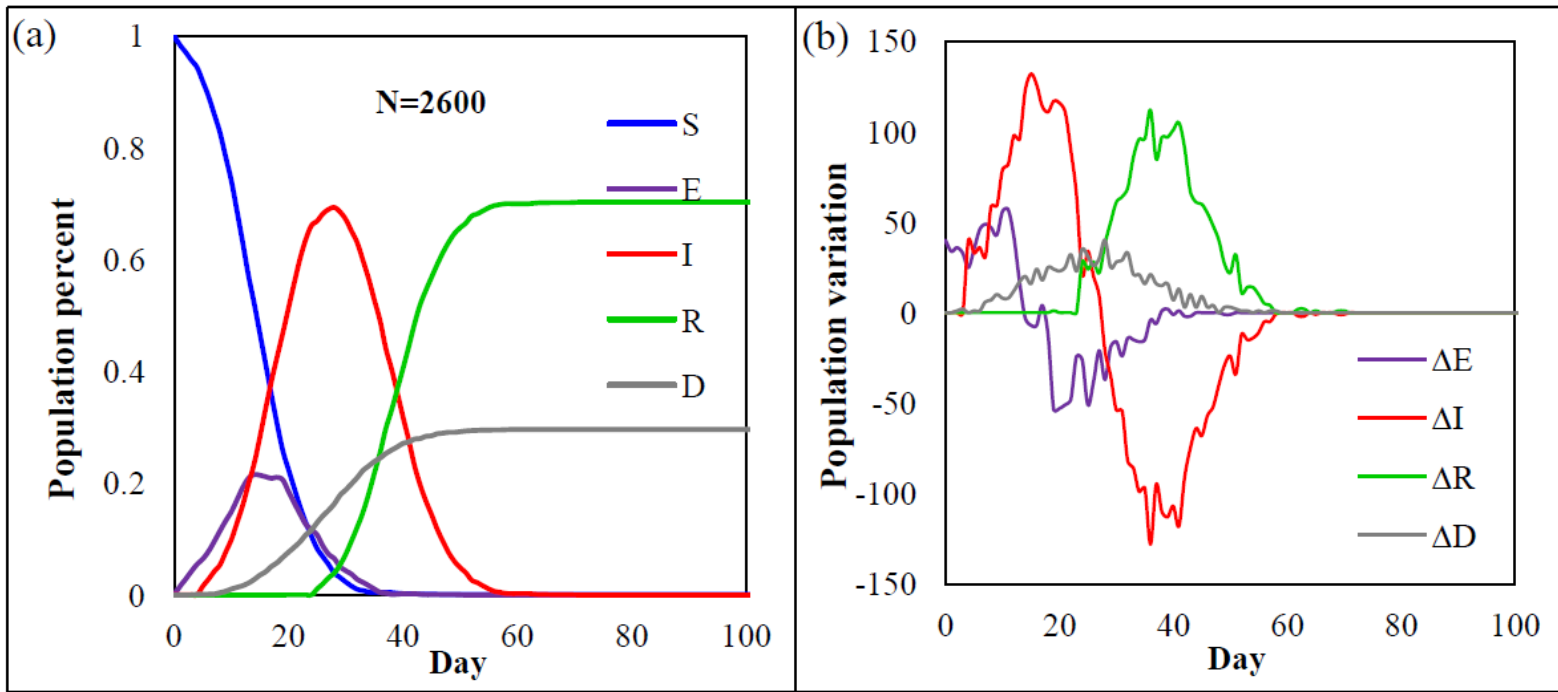

Figure 6: The effect of adding a public place in the system for the total population number of 2600 persons, (a) the percentage variations of the five populations during the time, (b) the population variation of $\mathrm{E}, \mathrm{I}, \mathrm{R}$ and $\mathrm{D}$ compartments per day. 


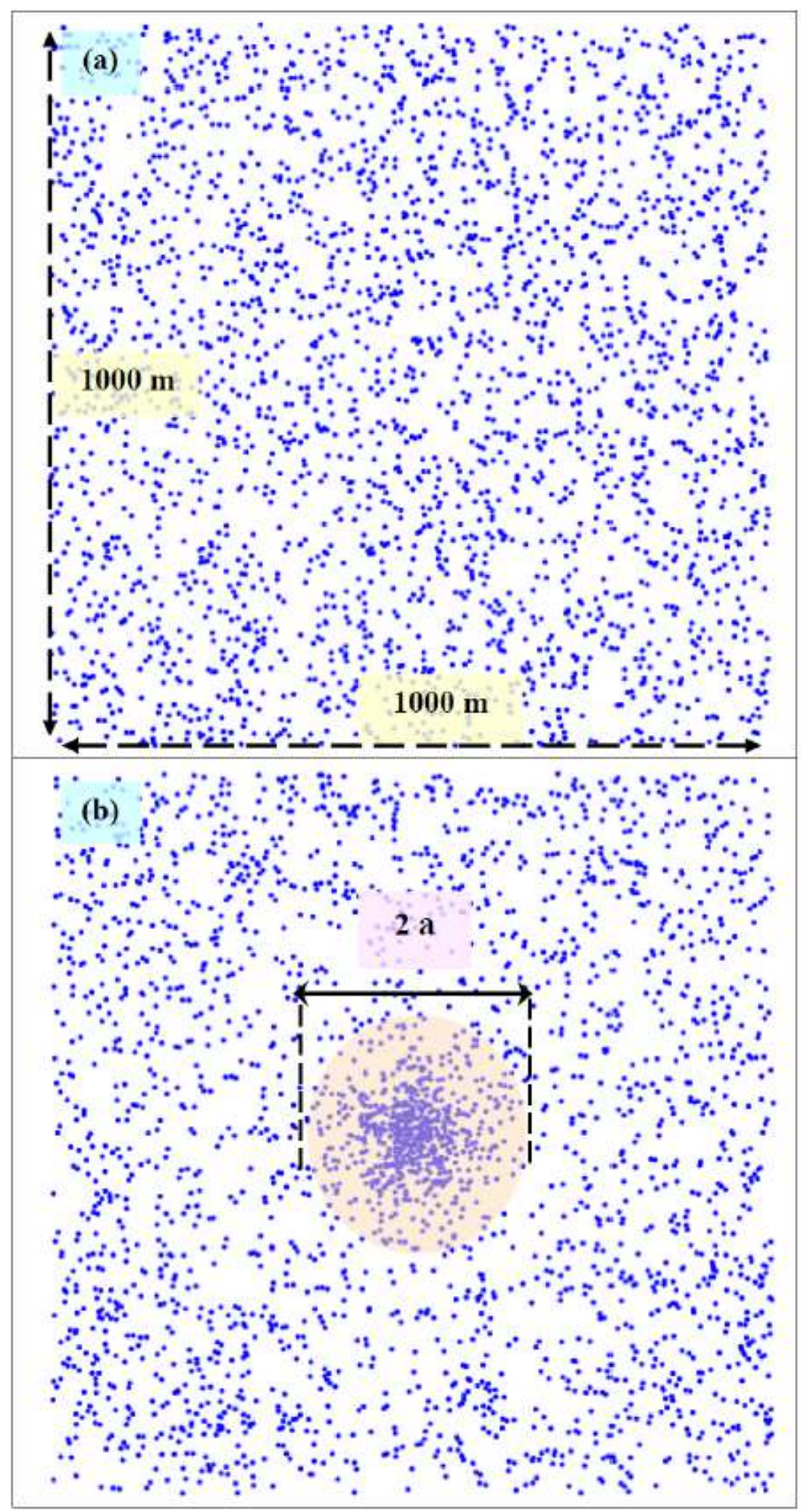

\section{Figure 1}

The initial arrangement of the number of 2600 people in the square simulation box of $1000 \times 1000 \mathrm{~m} 2$, (a) for the system without the crowded place, (b) for the system without the crowded place in the center of the simulation box. 


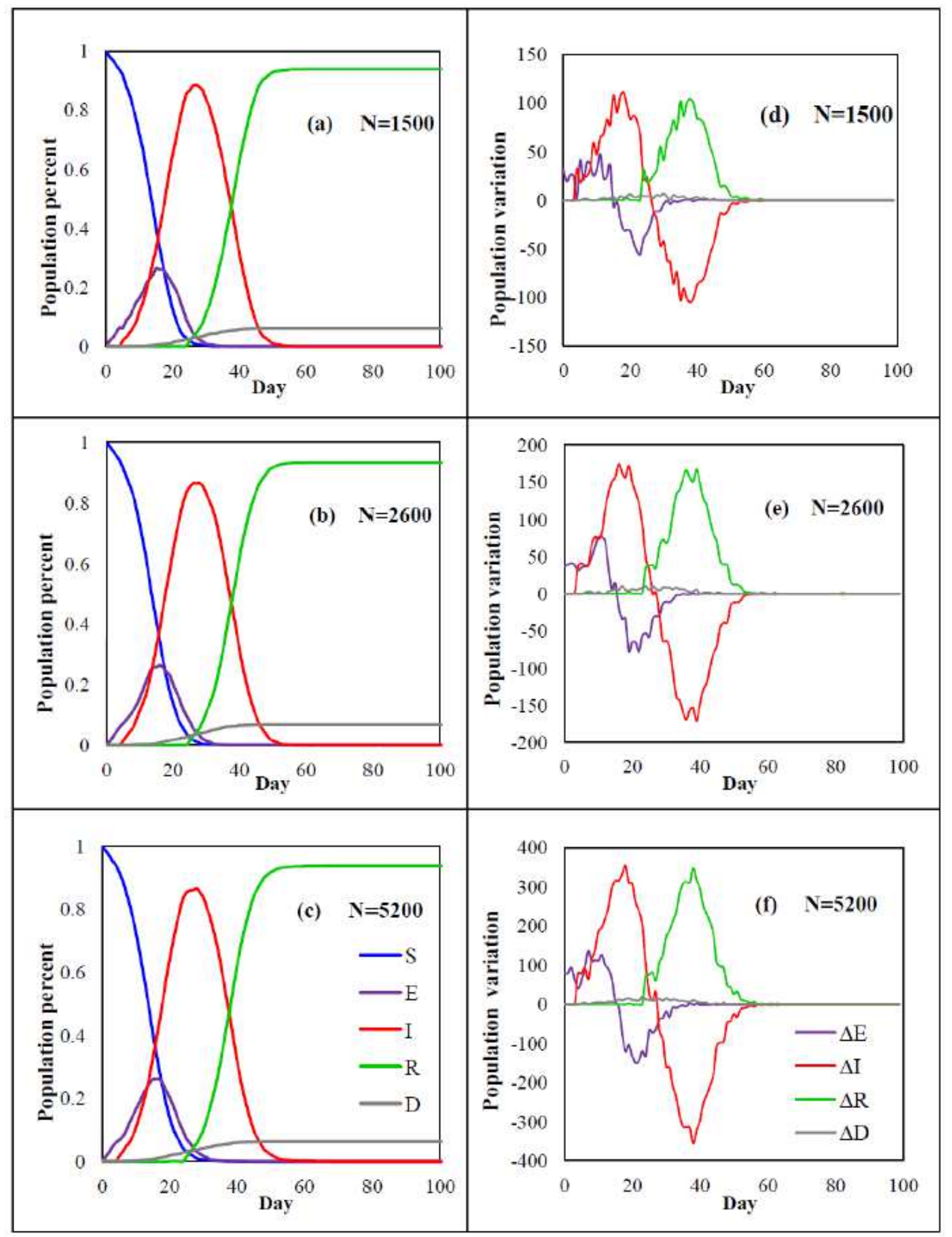

Figure 2

The percentage variations of the five populations during the time for the total population number of, (a) 1500 , (b) 2600 , and (c) 5200, and the population variation of E, I, R and D compartments per day for the total population number of, (d) 1500, (e) 2600, and (f) 5200, utilizing our model. 


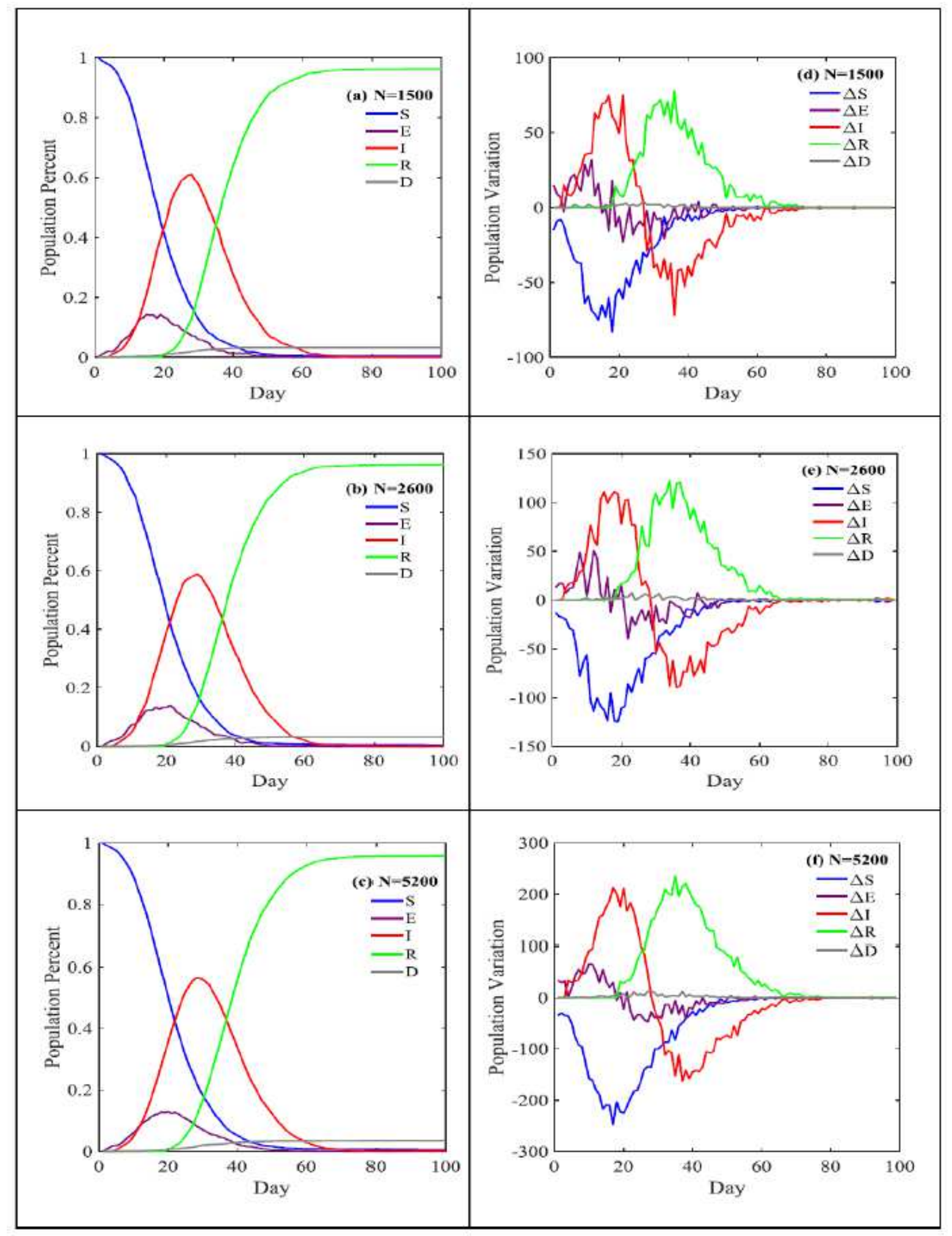

\section{Figure 3}

The percentage variations of the five populations during the time for the total population number of, (a) 1500 , (b) 2600, and (c) 5200, and the population variation of E, I, R and D compartments per day for the total population number of, (d) 1500 , (e) 2600 , and (f) 5200, utilizing classical SEIR model with a fixed square lattice network. 


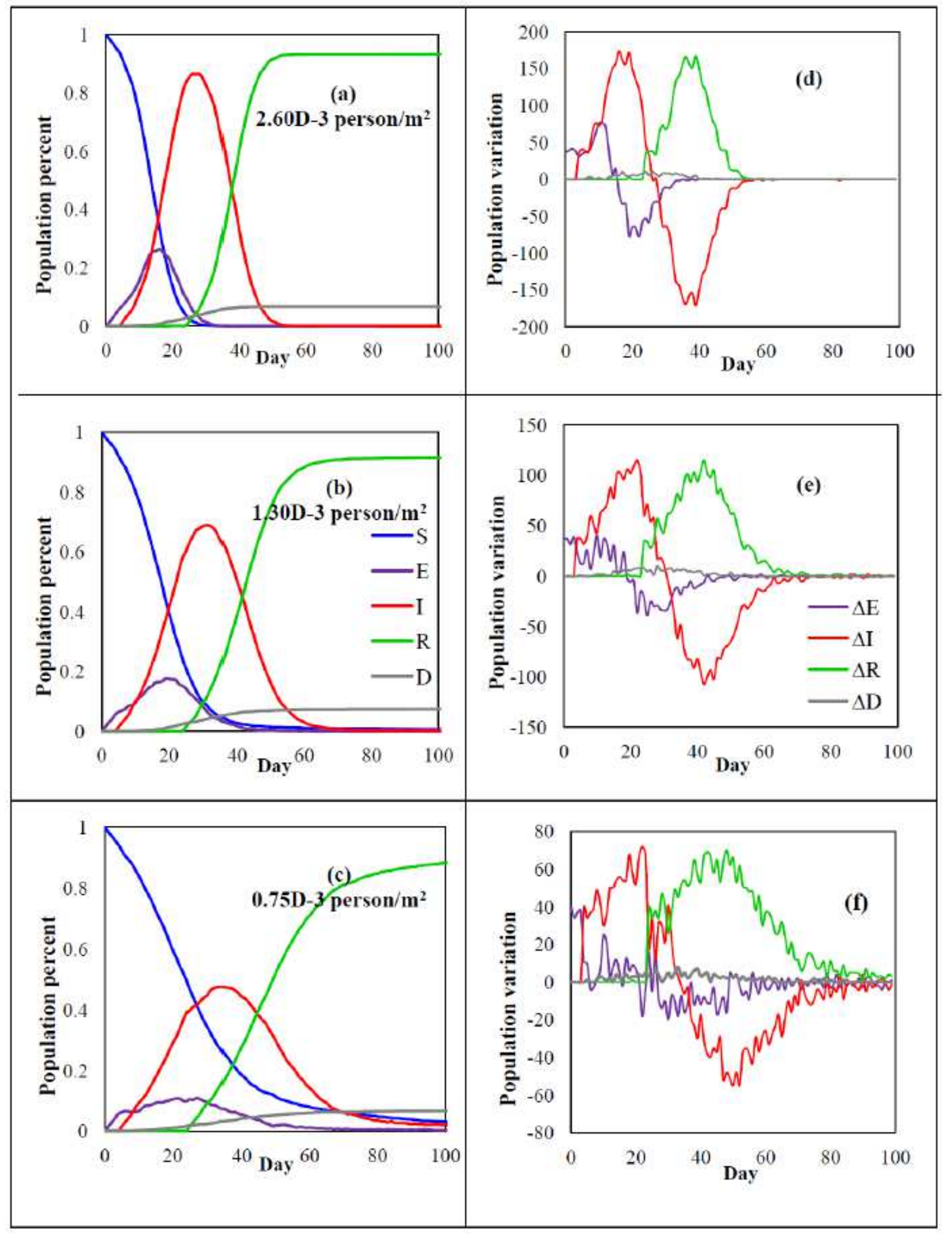

\section{Figure 4}

The percentage variations of the five populations during the time for the population number densities of, (a) 2.60D-3, (b) $1.30 \mathrm{D}-3$, and (c) $0.75 \mathrm{D}-3$ person $/ \mathrm{m} 2$, and the population variation of $\mathrm{E}, \mathrm{I}, \mathrm{R}$ and $\mathrm{D}$ compartments per day for the total population number of, (d) 2.60D-3, (e) 1.30D-3, and (f) $0.75 \mathrm{D}-3$ person/m2, utilizing our model. 


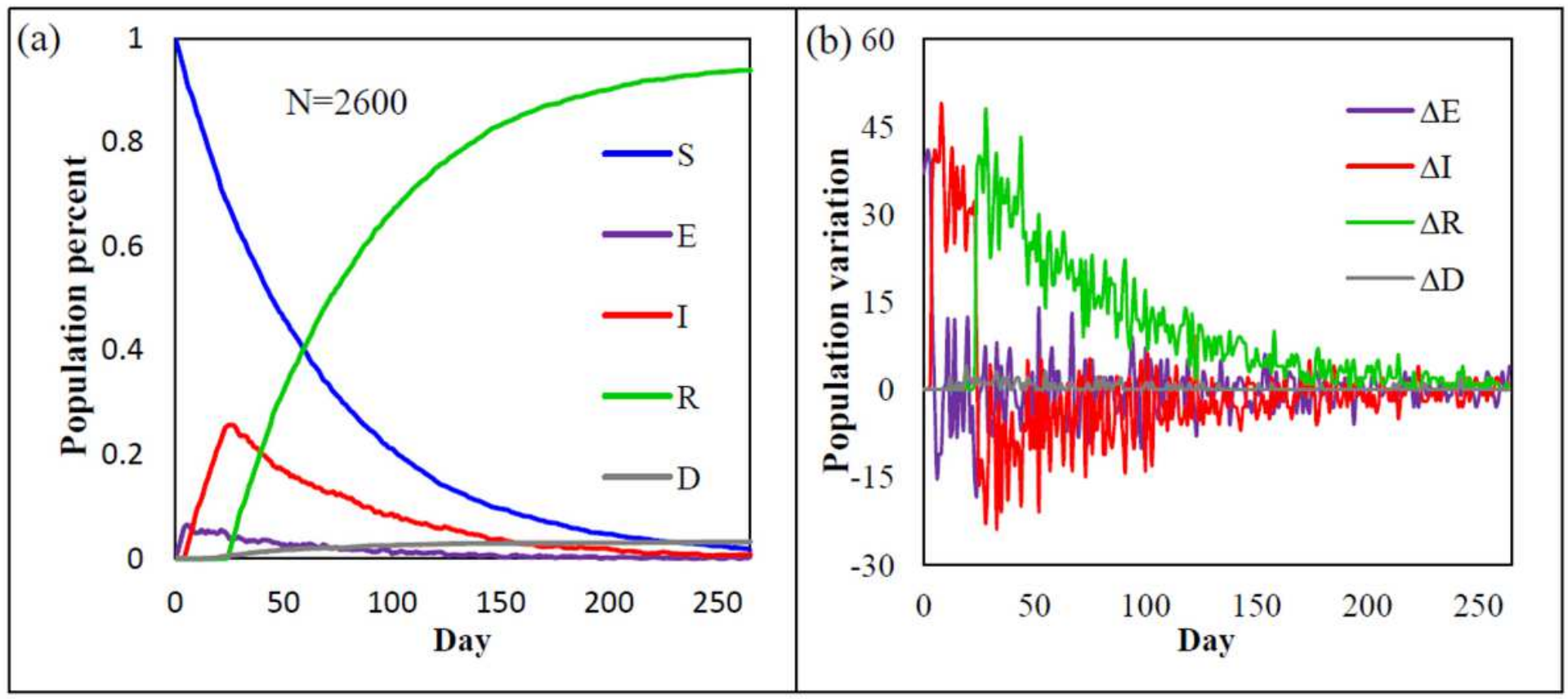

Figure 5

The effect of public health strategies for the total population number of 2600 persons, (a) the percentage variations of the five populations during the time, (b) the population variation of $E, I, R$ and $D$ compartments per day.

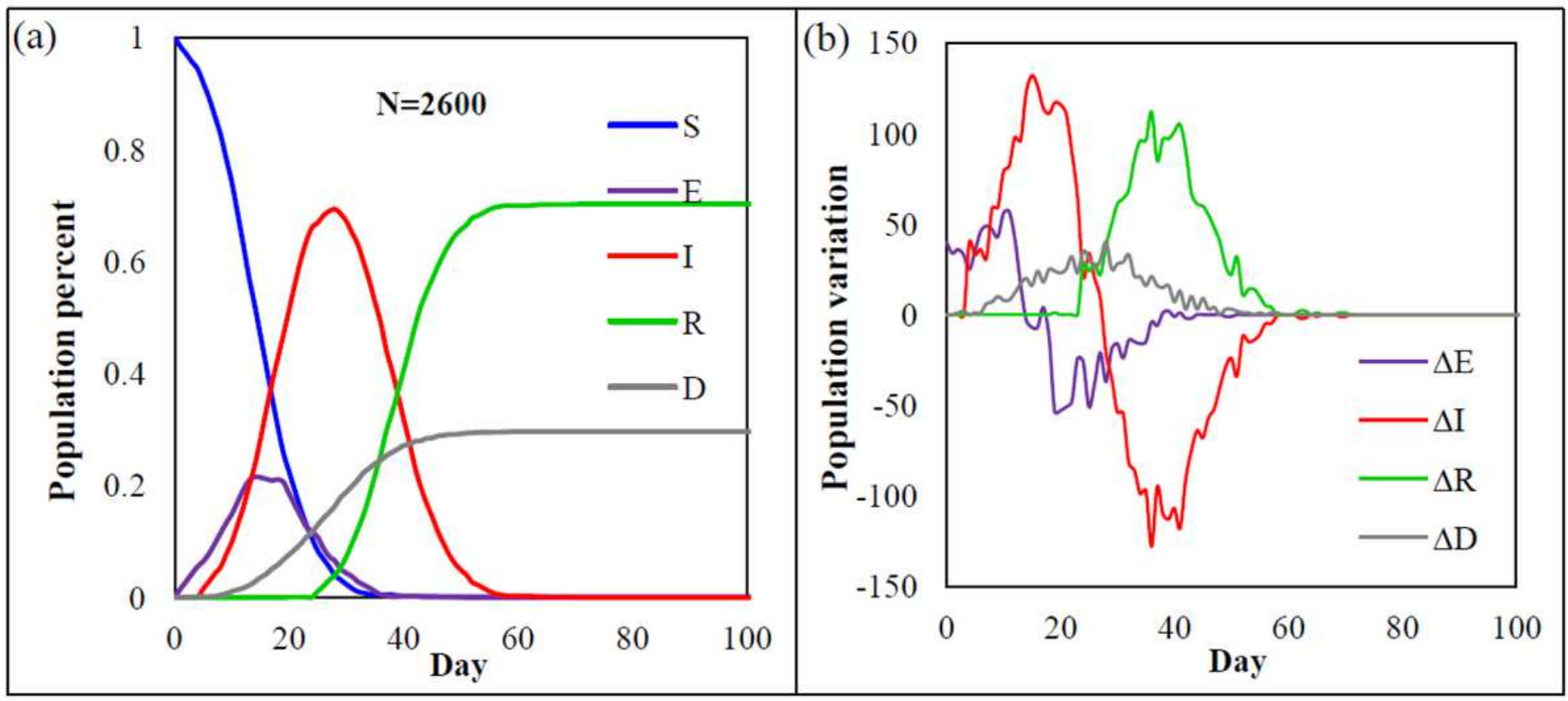

Figure 6

The effect of adding a public place in the system for the total population number of 2600 persons, (a) the percentage variations of the five populations during the time, (b) the population variation of $E, I, R$ and $D$ compartments per day. 